\title{
Postpartum Sinus Tachycardia
}

\author{
Nathan A. Keller \\ Department of Obstetrics and Gynecology, Albany Medical Center \\ 16 New Scotland Avenue, Second Floor, MC-74 Albany, New York, \\ Nathan A. Keller - Medical Doctor \\ No grants or financial supporters to report \\ kellern@mail.amc.edu
}

\begin{abstract}
Pregnancy is a time of immense physiological and immunological change. Perhaps no organ system undergoes more adaptations then the cardiovascular. Increased cardiac output, increased intravascular volume, hyper coagulability, and decreased systemic vascular resistance, are four essential alterations [1]. Additionally, a rise in pulse rate of ten to twenty beats per minute is usually seen. Further, these alterations do not immediately resolve after delivery. For instance, only approximately one week after delivery does blood volume return to its pre-pregnancy level with cardiac output reaching nonpregnant levels around 10 days post partum [2]. Assisting these alterations is an extensive diuresis process progressively removing extracellular water predominantly between post partum days two and five [2]. These alterations are paramount for the Obstetrician and Cardiologist alike to understand when evaluating the immediately post partum patient.
\end{abstract}

Sinus tachycardia may represent the most common rhythm disturbance [3]. Sinus tachycardia has traditionally been defined as a rate exceeding 100 beats per minute arising from the sino-atrial node and in many cases representing a normal sympathetic tone increase or a parasympathetic tone decrease [3]. This rhythm disturbance represents an eclectic group of disorders and an exact diagnosis of the cause is often challenging [3]. Further complicating matters is the fact that a large fraction of patients with sinus tachycardia have no specific symptoms [3]. When symptomatology does occur,palpitations, shortness of breath, chest pain, dizziness, and pre-syncope are common [3]. Some common causes include but are not limited to volume decrease, anemia, sepsis, fever, pain, pulmonary embolism, and hyperthyroidism, among many others [3]. Unfortunately, the normal cardiovascular physiological changes of pregnancy make diagnosing the cause of this rhythm disturbance in the immediate post partum period even more challenging.

The immune system is also critically affected by pregnancy. Specifically, pregnancy and the immediate postpartum period, are times of relative immune compromise creating a milieu of physiologic and immunologic change that not only can be inducive to infectious etiology, but can help mask often dangerous infections.For instance, it is theorized that pregnancy is a state of systemic immune suppression, an adaptation to inhibit rejecting the fetus as foreign [4]. Further, although likely far more complicated, it has been thought that normal pro-inflammatory states are decreased in pregnancy while anti-inflammatory states are increased [5]. These immune system adaptations lend the pregnant woman to often increased susceptibility to potentially highly morbid infections.

Perhaps no modern day infection can be as elusive, yet as dangerous, as Group A Streptococcus (GAS). GAS, most commonly Streptococcus pyogenes (S. pyogenes), is an entity that is most well known for the colonization of children and young adults in the oropharynx causing "strept throat" [6]. Spread of this pathogen is predominantly through respiratory droplets and acquisition is highest in daycare facilities and other crowded areas with children and young adults [6]. Additionally, acquisition is seasonal with the maximum times of spread in the winter months [6]. Unfortunately, $S$. pyogenes, has multiple virulence factors that allow the organism to become extremely dangerous and potentially lethal most often through the processes of necrotizing fasciitis or streptococcal toxic shock syndrome. For instance, in women who develop necrotizing fasciitis involving S. pyogenes, it is estimated that the mortality rate approaches 50\% [6]. Streptococcal toxic shock syndrome poses distinct challenges for physicians which are only confounded more by the physiological changes of pregnancy. For instance, nonspecific symptoms such as malaise, fevers, diarrhea, and chills are often the presenting signs in this highly morbid infection [6]. Further, immunocompromised patients such 
as those with HIV, diabetes, and cancer are at especially high risk [6]. Unfortunately, when blood cultures are positive in patients with Streptococcal toxic shock syndrome, mortality is close to $40 \%$ [6].

The reasons surrounding the high virulence of Group A Streptococcus are many. Perhaps most important is Group A streptococcus' ability to produce pyrogenic exotoxins causing delayed hypersensitivity, cytotoxicity, and B-cell immunosuppression [6]. The bacteria also contain an antiphagocytic capsule and an M protein instrumental in cell adhesion [6]. Streptolysin S and O further function in lysing multiple cells including leukocytes, platelets, and erythrocytes [6]. These factors make group A Streptococcus exceptionally dangerous when skin colonization occurs with consequent invasion to deeper tissues as is possible during breaks in the skin. In our case, an episiotomy site likely represented the point of entry of this potentially devastating infection.

Recently our institution received a patient from an outside hospital that challenged numerous providers, Cardiologists, ER staff, and Obstetricians and Gynecologists alike. The original full case report is referenced here [7]. Below is provided a summary of this patient's hospital course. If more specific details about the hospital course are desired please reference the source provided [7].

In summary,a Caucasian woman in her mid thirties presented to our facility four days after a vaginal delivery with palpitations and tachycardia. The recent delivery was complicated only by an episiotomy. The patient had no history of tachycardia and no other significant history. On post partum day (PPD) \#1 she had presented to an outside hospital with shortness of breath and was found to be in sinus tachycardia to the mid 120s/minute with normalchest $\mathrm{x}$-ray andnegative pulmonary CT scans.The following day sherepresented to another ER complainingagain of palpitations and was found to have a heart rate in the mid 130s per minute with a sinus tachycardia. Laboratory testing was again otherwise normal. Since an echocardiogram showed no abnormalities, the patient was sent home with a script for metoprolol. Three days later she came to yetanother emergency room with yet more palpitations and dyspnea superimposed withwhole body soreness and a sinus tachycardia in the 130s per minute. She was still afebrile with no uterine symptomatology. Chest CT, abdominal CT, and abdominal ultrasound were normal. Unfortunately, speculum and pelvic examinations were not performed. Upon being transported to our hospital, the patient was sent to the Surgical Intensive Care Unit secondary to concern for sepsis. Vitalsincluded heart rate of 139 per minute, respirations of 30 per minute, and blood pressure of 121/66 mmHg. EKG again showed sinus tachycardia (Figure 1). Piperacillin-tazobactam (zosyn) was initiated for broad spectrum coverage of a presumed sepsis. Abdominal examination revealed a few centimeters of erythema approximately $5 \mathrm{~cm}$ above the pubic symphisis in the midline. Additionally, the labia minora and majorahad extensive bilateral erythema and two small ulcerative lesions were seen on the cervix with purulent yellow discharge present. At that time Toxic Shock Syndrome (TTS) related to Group AStreptococcus was suspected.

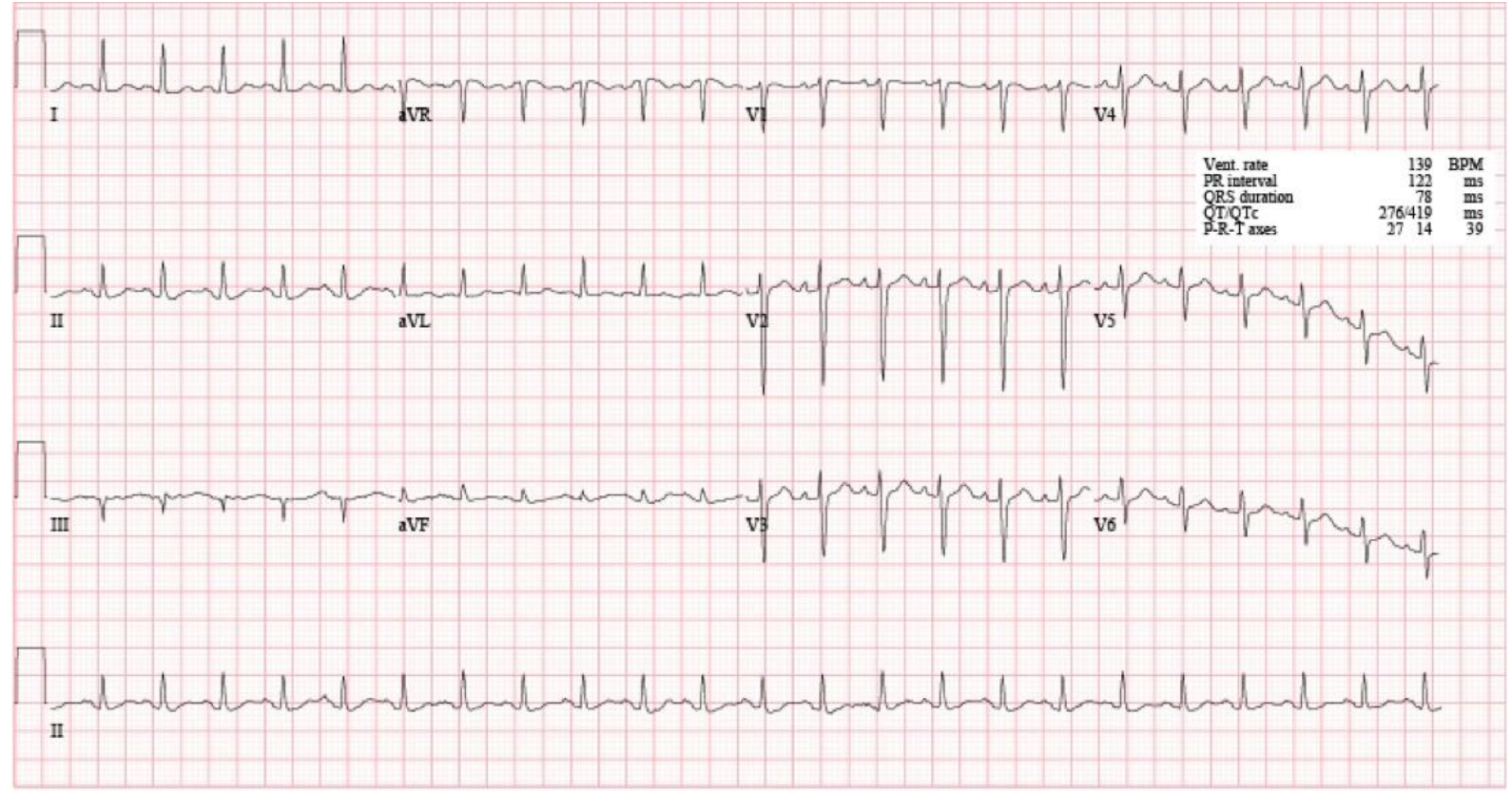

Figure1. Sinus tachycardia on transfer to tertiary care center 
This was later confirmed by urine and blood cultures. Our team also learned that the patient's young toddler and husband both had had $S$. pyogenesduring the past four weeks. Ultimately, the patient required a total abdominal hysterectomy and bilateral salpingo-oophorectomy, multiple blood transfusions, vasopressors, extensive antibiotic therapy, and intubation. She later developed an acute kidney injury and an infection in her forearm, which required a decompressive fasciotomy and carpel tunnel release (Figure 2).

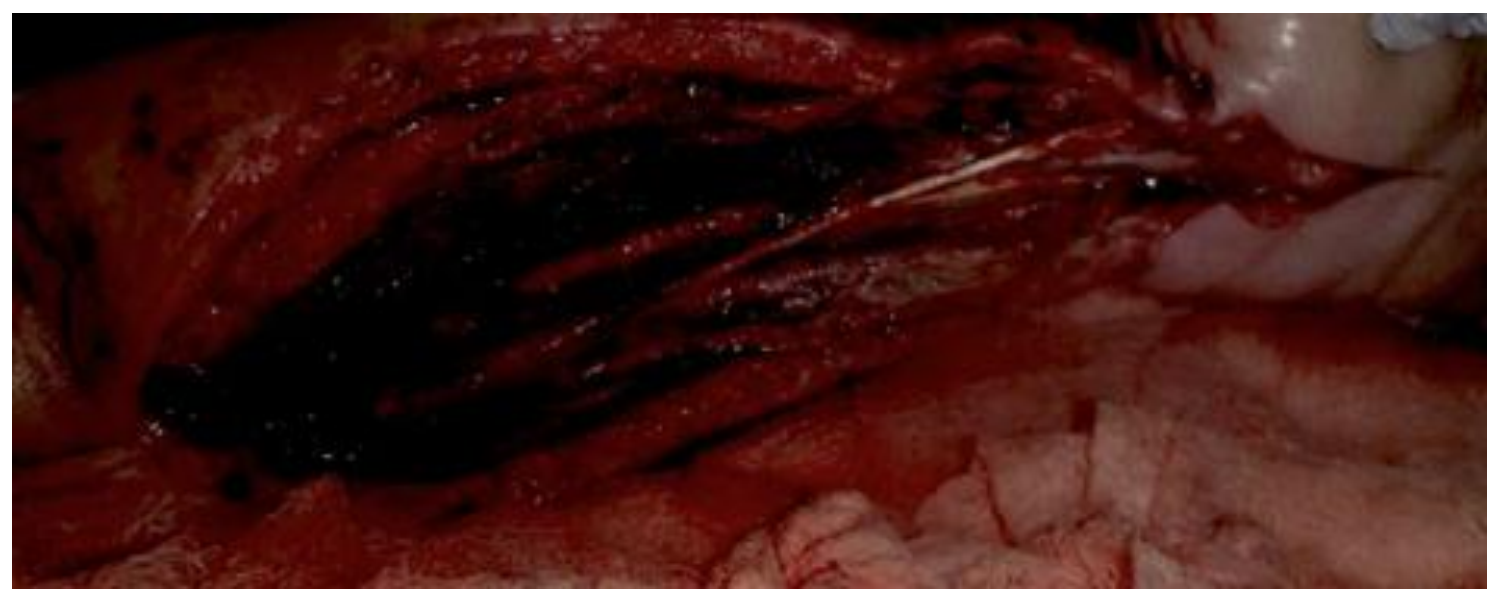

Figure2. Left forearm status post decompressive fasciotomy of the flexor and extensor compartments as well as carpel tunnel release with median nerve neurolysis.

This case offers many important lessons for the cardiologist, emergency room physician, and obstetrician alike. First, S. pyogenescan be potentially extremely morbid having an estimated case fatality ratio of fifteen to twenty percent skyrocketing to forty to sixty percent in the presence of septic shock [8]. Further, this makes GAS one of the most lethal bacterial pathogens known to mankind with postpartum women incurring a 20-fold increased incidence of GAS[8]. Despite this tremendous risk to pregnant and postpartum women it is estimated that fifteen to twenty percent of school aged children may carry this bacterial strain [9]. This specific case shows that postpartum GAS can have very nonspecific symptomatology, in this instancenew onset sinus tachycardia accompanied by persistent postpartum palpitations. Thus physicians, and especially a consulting Cardiologist, are in a unique position to suggest and ultimately lead to identification of a diagnosis which may have eluded the primary team. This fact cannot be overemphasized especially since the initial complaint of sinus tachycardia in the post partum period may be unlikely to facilitate a gynecology consult.

Our case illustrates the early diagnosis is critical yet challenging. Unfortunately, in this case sinus tachycardia with palpitations of unknown etiology seemed to direct many physicians towards cardiac and pulmonary sources. To the contrary,a potential infectious etiology received much less attention. Given the virulence of GAS infection and the markedly increased risk in pregnancy, effective screening and treatment at first glance would appear to be very valuable. Unfortunately, the literature does not validate this thought. For instance, screening for GAS during pregnancy is not practicalas GAS colonization of the vagina during pregnancy is very rare, as low as $0.03 \%$ in one study [10]. Further, in this study these identified individuals received no treatment yet did not develop a clinically recognizable infection [10]. This signifies that the clinical utility of screening for GAS infection in pregnancy has not been realized and the relationship between colonization and symptomatic infection still remains incompletely understood.

It is worth mentioning that in busy emergency room settings, an Obstetrician may not even be consulted for a complaint of postpartum tachycardia. This puts the consulting Cardiologist in a unique position to help make the diagnosis. A suggestion could be brought forward thatwhen evaluating a postpartum tachycardic patient, a brief screen by the Cardiologist for potential contacts diagnosed with or possessing signs of $S$. pyogenes could be very valuable. Further, asking about vaginal tears, episiotomies, rupture of membranes, and method of delivery is significant as well as both alterations in cutaneous and mucosal barriers and increased vaginal $\mathrm{pH}$ from amniotic fluid create an environment amenable to GAS propagation [11]. While seemingly relatively minor and straight forward, given the morbidity and mortality associated with delayed diagnosis of GAS in conjunction with the absence of effective screening, such questioning may have profound benefits especially in the situation where an Obstetrician is not consulted to see the patient. Further, if such risk factors are 
identified by the Cardiologist suggesting a pelvic exam, preferably by an Obstetrician, is highly encouraged and could potentially be life saving.

In conclusion, Group a Streptococcal infections in the post partum period can have varied and subtle presentations, yet especially devastating consequences if undetected. Persistent postpartum sinus tachycardia may be the initial presenting symptom, a situation in which a Cardiologist may be consulted but an Obstetrician may not. In this situation, cognizance on the part of the Cardiologist with additional questioning about contacts with a known diagnosis of, or signs/symptoms suggestive of,Streptococcus pyogenes could be immensely valuable. Additionally, basic questioning about recent lacerations, episiotomies, premature rupture of membranes, and mode of delivery could further heighten suspicion and ultimate diagnosis of this potentially morbid condition. Finally, when such risk factors are identified suggesting a gynecological consultation with thorough pelvic exam could be life saving.

\section{ACKNOWLEDGMENT}

The authors would like to acknowledge the Surgical Intensive Care Unit team which provided high quality care to this patient throughout her hospitalization.

\section{REFERENCES}

[1] Queenan JT, Spong CY, Lockwood CJ (2012). Queenan's Management of High-Risk Pregnancy; An Evidence-Based Approach. Sixth Edition. Wiley-Blackwell A John Wiley \& Sons Publication, West Sussex, UK.

[2] Cunningham FG, Leveno KJ, Bloom SL, Hauth JC, Rouse DJ, Spong CY (2010). Williams Obstetrics. Twenty Third Edition. The McGraw-Hill Companies, New York, NY.

[3] Yusuf S, Camm J (2005). Deciphering the Sinus Tachycardias. Clinical Cardiology. 28: 267-276.

[4] Creasy RK, Resnik R, Iams JD, Lockwood CJ, Moore TR (2009). Creasy \& Resnik's MaternalFetal Medicine. Sixth Edition. Saunders Elsevier, Philadelphia, Pennsylvania.

[5] Johnson CT, Hallock JL, Bienstock JL, Fox HE, Wallach EE (2015). The Johns Hopkins Manual of Gynecology and Obstetrics. Fifth Edition. Wolters Kluwer, China.

[6] Murray PR, Rosenthal KS, Kobayashi GS, Pfaller MA (2002). Medical Microbiology. Fourth Edition. Mosby, A Harcourt Health Sciences Company, St. Louis, Missouri.

[7] Keller NA, Guan X, Wiczulis A, Burcher P (2015). Unexplained persistent postpartum palpitations and tachycardia due to Group A Streptococcus. BMC Research Notes. 2015;8:731. doi:10.1186/s13104-015-1739-y.

[8] Rimawi BH, Soper DE, Eschenbach DA (2012). Group A streptococcal infections in obstetrics and gynecology. Clin Obstet Gynecol. 55:864.

[9] Martin JM, Green M, Barbadora KA, et al (2004). Group A streptococci among school-aged children; clinical characteristics and the carrier state. Pediatrics. 114: 1212-1219.

[10] Mead P, Winn WC (2000). Vaginal-Rectal Colonization With Group A Streptococci in LatePregnancy. Infect Dis in Obstetrics and Gynecology. 8:217-219.

[11] Bisno A, Stevens D (2005). Streptococcal infections of skin and soft tissue. New England Journal of Medicine. 334:240-246.

This article is based on a previous article published by the same corresponding author:

Keller NA, Guan X, Wiczulis A, Burcher P. Unexplained persistent postpartum palpitations and tachycardia due to Group A Streptococcus.

BMC Research Notes. 2015; 8:731. doi:10.1186/s13104-015-1739-y. 